\title{
\begin{tabular}{l|l} 
Mibraries & DSpace@MIT
\end{tabular}
}

\author{
MIT Open Access Articles
}

Young and old genetically heterogeneous HET3 mice on a rapamycin diet are glucose intolerant but insulin sensitive

The MIT Faculty has made this article openly available. Please share how this access benefits you. Your story matters.

Citation: Lamming, Dudley W., Lan Ye, Clinton M. Astle, Joseph A. Baur, David M. Sabatini, and David E. Harrison. "Young and Old Genetically Heterogeneous HET3 Mice on a Rapamycin Diet Are Glucose Intolerant but Insulin Sensitive." Aging Cell 12, no. 4 (June 7, 2013): 712-718. ( 2013 John Wiley \& Sons Ltd and the Anatomical Society

As Published: http://dx.doi.org/10.1111/acel.12097

Publisher: Wiley Blackwell

Persistent URL: http://hdl.handle.net/1721.1/96741

Version: Final published version: final published article, as it appeared in a journal, conference proceedings, or other formally published context

Terms of use: Creative Commons Attribution 


\section{Young and old genetically heterogeneous HET3 mice on a rapamycin diet are glucose intolerant but insulin sensitive}

Dudley W. Lamming, ${ }^{1,2,3,4,5}$ Lan Ye, ${ }^{6}$ Clinton M. Astle, ${ }^{7}$ Joseph A. Baur, ${ }^{6}$ David M. Sabatini ${ }^{1,2,3,4,5}$ and David E. Harrison ${ }^{7}$

${ }^{1}$ Whitehead Institute for Biomedical Research, Cambridge, MA 02142, USA ${ }^{2}$ Department of Biology, MIT, Cambridge, MA 02139, USA

${ }^{3}$ Howard Hughes Medical Institute, MIT, Cambridge, MA 02139, USA

${ }^{4}$ Broad Institute of Harvard and MIT, Seven Cambridge Center, Cambridge, MA 02142, USA

${ }^{5}$ The David H. Koch Institute for Integrative Cancer Research at MIT, Cambridge, MA 02139, USA

${ }^{6}$ Department of Physiology, Institute for Diabetes, Obesity, and Metabolism, Perelman School of Medicine, University of Pennsylvania, Philadelphia, PA 19104, USA

${ }^{7}$ The Jackson Laboratory, Bar Harbor, ME 04609, USA

\section{Summary}

Rapamycin, an inhibitor of the mechanistic target of rapamycin (mTOR) signaling pathway, extends the life span of yeast, worms, flies, and mice. Interventions that promote longevity are often correlated with increased insulin sensitivity, and it therefore is surprising that chronic rapamycin treatment of mice, rats, and humans is associated with insulin resistance (J Am Soc Nephrol., 19, 2008, 1411; Diabetes, 00, 2010, 00; Science, 335, 2012, 1638). We examined the effect of dietary rapamycin treatment on glucose homeostasis and insulin resistance in the genetically heterogeneous HET3 mouse strain, a strain in which dietary rapamycin robustly extends mean and maximum life span. We find that rapamycin treatment leads to glucose intolerance in both young and old HET3 mice, but in contrast to the previously reported effect of injected rapamycin in C57BL/6 mice, HET3 mice treated with dietary rapamycin responded normally in an insulin tolerance test. To gauge the overall consequences of rapamycin treatment on average blood glucose levels, we measured HBA1c. Dietary rapamycin increased HBA1c over the first 3 weeks of treatment in young animals, but the effect was lost by 3 months, and no effect was detected in older animals. Our results demonstrate that the extended life span of HET3 mice on a rapamycin diet occurs in the absence of major changes in insulin sensitivity and highlight the importance of strain background and delivery method in testing effects of longevity interventions. Key words: aging; glucose tolerance; heterogeneous mice; insulin tolerance; rapamycin.

\section{Introduction}

Calorie restriction $(\mathrm{CR})$, an intervention in which total caloric intake is reduced while maintaining nutrition, extends the life span of

\section{Correspondence}

David E. Harrison, The Jackson Laboratory, 600 Main Street, Bar Harbor, ME 04609, USA. Tel.: 207-288-6357; fax: 207-288-6077; e-mail: david.

harrison@jax.org

Accepted for publication 24 April 2013 yeast, worms, flies, and mammals, including mice, rats, dogs, and primates. A CR diet [and often even diet restriction (DR), in which amounts of the diet are simply reduced] prevents or delays the incidence of numerous age-related diseases, including type 2 diabetes. Accordingly, there is significant interest in identifying small molecules that mimic the beneficial effects of a CR diet. The National Institute on Aging Interventions Testing Program (ITP) tests the effects of anti-aging interventions on mouse longevity at three independent sites, using genetically heterogeneous HET3 mice. HET3 mice are the F2 progeny of (BALB/CJ $\times$ (57BL/6J) mothers and $(\mathrm{C} 3 \mathrm{H} / \mathrm{HeJ} \times \mathrm{DBA} / 2 \mathrm{~J})$ fathers and provide a heterogeneous yet reproducible genetic background in which the causes of age-related mortality will be varied (Flurkey et al., 2010). To date, the molecule in this program that demonstrates the most robust extension of both average and maximum life spans is rapamycin (Harrison et al., 2009; Miller et al., 2011).

Rapamycin is a specific inhibitor of the mechanistic target of rapamycin (mTOR), a protein kinase that is found in two complexes with distinct cellular targets. MTORC1, the canonical target of rapamycin, regulates many processes related to growth, including ribosomal biogenesis, cap-dependent translation, and autophagy. mTORC2, which regulates Akt, SGK, and PKC $\alpha$ activity, is resistant to acute treatment with rapamycin. However, chronic treatment disrupts mTORC2 signaling in some cell lines (Sarbassov et al., 2006).

We recently showed that chronic treatment with rapamycin disrupts mTORC2 signaling in vivo (Lamming et al., 2012). Rapamycin treatment of young BALB/c mice (Cunningham et al., 2007) and C57BL/6 mice (Lamming et al., 2012) for 2-3 weeks leads to glucose intolerance and hepatic insulin resistance, mediated by the disruption of mTORC2 (Lamming et al., 2012). Chronic rapamycin treatment of C57BL/6 mice may also lead to mTORC1-mediated skeletal muscle insulin resistance (Cunningham et al., 2007; Blattler et al., 2012). Young Sprague-Dawley rats chronically treated with rapamycin develop glucose intolerance and insulin resistance and have increased hepatic gluconeogenesis (Houde et al., 2010). Similar results have also been observed in humans treated with rapamycin, with kidney transplant patients receiving rapamycin displaying increased insulin sensitivity, and the 3-year incidence of diabetes increasing to nearly $22 \%$ in rapamycin-treated patients, compared with $16-19 \%$ in patients receiving alternative treatment (Teutonico et al., 2005; Johnston et al., 2008).

Treatments that increase life span frequently also increase insulin sensitivity, as observed in mice on a CR diet as well as in the longlived Ames and Snell dwarf mice, as well as many genetically modified mice (Dominici et al., 2002; Selman et al., 2009; Foukas et al., 2013). While certain genetically modified mice strains have both increased insulin resistance and increased longevity (Selman et al., 2008), decreased insulin sensitivity in both mice and humans 
is more typically associated with decreased life span (Hogan et al., 2003; Baur et al., 2006). It is therefore fascinating that rapamycin decreases glucose tolerance and insulin sensitivity in the context of increased life span.

However, studies of the effects of rapamycin on glucose homeostasis have been performed exclusively in young, inbred rodent strains given rapamycin by intraperitoneal injections for only a few weeks. In contrast, major longevity studies using rapamycin have been performed in genetically heterogeneous HET3 mice, with dietary rapamycin treatment beginning at 9 or 20 months of age and persisting until death. Thus, to better understand the paradoxical effects of rapamycin on diabetic phenotypes, we examined glucose homeostasis and insulin sensitivity in both young and old HET3 mice treated with dietary rapamycin for either 3 weeks or 3 months.

\section{Results}

Rapamycin treatments were scheduled, so our analyses below of 3-week and 3-month treatments were done when HET3 mice were 6 or 21 months of age. Fasted weights taken just before testing showed no statistically significant differences, although young mice fed rapamycin tended to be lighter than age-matched controls, while old mice fed rapamycin tended to be heavier than agematched controls.

Rapamycin significantly decreased glucose tolerance in both young (Fig. 1A) and old (Fig. 1B) HET3 mice. The length of rapamycin treatment did not significantly affect the area under the curve (Fig. 1C), although the area tended to be higher in young mice given rapamycin for 3 months. These results are consistent with previous findings that rapamycin treatment of C57BL/6 mice and Sprague-Dawley rats increases gluconeogenesis and induces hepatic insulin resistance (Houde et al., 2010; Lamming et al., 2012).

We also tested the response of rapamycin-fed mice to pyruvate, a key substrate for gluconeogenesis. Impaired tolerance to pyruvate indicates a failure to suppress hepatic gluconeogenesis. We found that young HET3 mice treated with rapamycin for 3 months (Fig. 1D), as well as old HET3 mice treated with rapamycin for either 3 weeks or 3 months (Fig. 1E), had significantly decreased pyruvate tolerance (Fig. 1F).
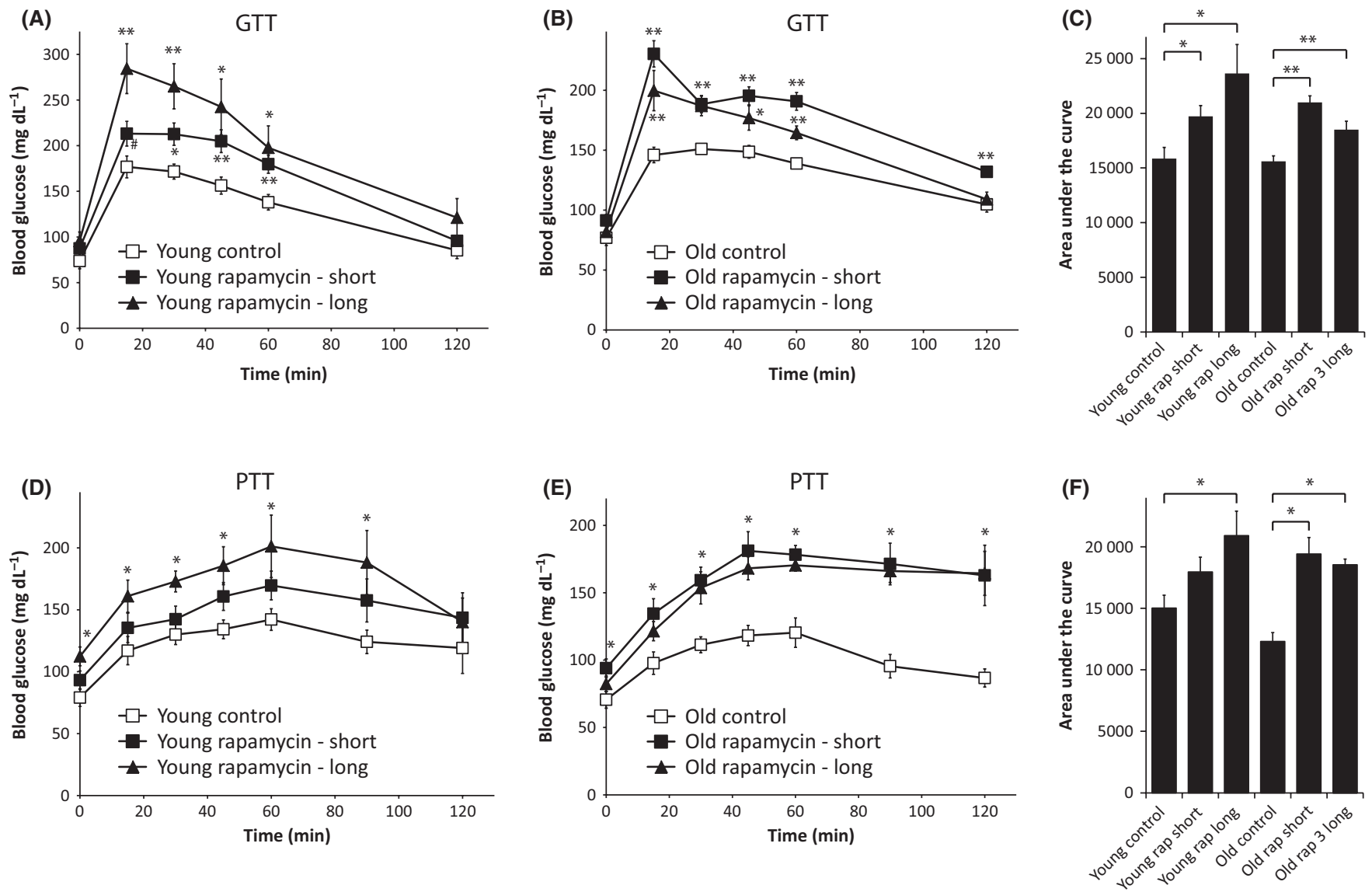

Fig. 1 Rapamycin treatment results in glucose and pyruvate intolerance in HET3 mice. Glucose tolerance test on (A) young (6-month-old) and (B) old (21-month-old) mice treated with rapamycin for short ( 3 weeks) or long ( 3 months) periods. (C) Quantitation of area under the curve of glucose tolerance tests in $(A)$ and (B). ( $A-C: * * P<0.01$, $* P<0.04,{ }^{\#} P<0.07$ vs. control mice, two-tailed $t$-test). Pyruvate tolerance was tested 2 weeks after glucose tolerance in the same mice and is decreased in (D) young mice treated with rapamycin for 3.5 months ( $* P<0.05$, young rapamycin-long vs. control mice, two-tailed $t$-test) and in (E) old mice treated with rapamycin for 5 weeks or 3.5 months ( $* P<0.05$, old rapamycin groups vs. old control mice, two-tailed $t$-test; at $t=0, P<0.05$ for rapamycin long vs. control only). ( $F)$ Quantitation of area under the curve of pyruvate tolerance tests in D and E. (D-F: $* P<0.05$, two-tailed $t$-test). 
In C57BL/6 mice and Sprague-Dawley rats, rapamycin treatment significantly induces insulin resistance, as measured by an insulin tolerance test (ITT) (Houde et al., 2010; Blattler et al., 2012; Lamming et al., 2012). Surprisingly, and in contrast to these results, we found that dietary rapamycin did not induce insulin resistance in HET3 mice as measured by ITT in either young (Fig. 2A) or old (Fig. 2B) mice, even when given to mice over a 3-month period. Interestingly, Fok et al., (2012) have recently reported that dietary rapamycin caused glucose intolerance in the absence of any effect on insulin tolerance in C57BL/6 mice, suggesting that the delivery method, rather than the strain, may be the major determinant of whether mice develop overt insulin resistance. In concurrence with our previous study in C57BL/6 mice, HET3 mice treated with rapamycin are hyperglycemic after refeeding and when young and treated with rapamycin for 3 months, also display fasting hyperglycemia (Fig. 2C).

Rapamycin has been reported to negatively impact glucosestimulated insulin release by pancreatic beta cells in C57BL/6 mice (Yang et al., 2012). We observed no significant differences in insulin levels between control mice and rapamycin-treated mice in either the fasting or refed state, in either young or old mice (Fig. 2D). We performed a glucose-stimulated insulin secretion (GSIS) assay (Fig. 2E). In contrast to the results reported in C57BL/6 mice, rapamycin caused no significant decrease in insulin levels following
(A)

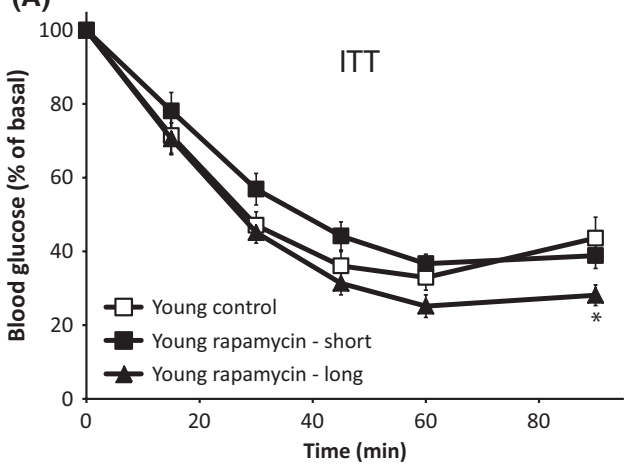

(B)

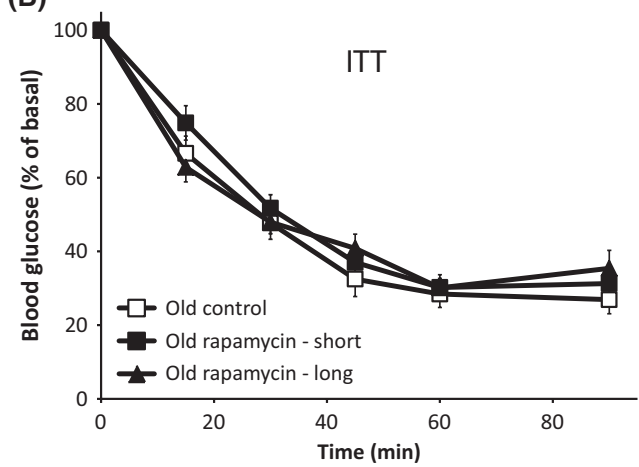

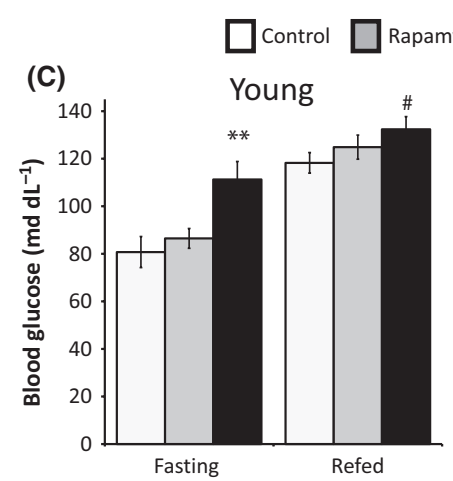

\section{(n)}

Rapamycin - long

\begin{abstract}
Old
\end{abstract}

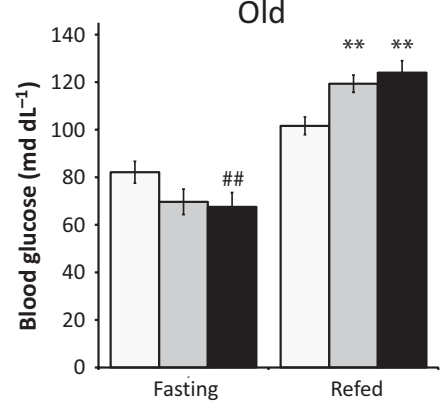

(D)
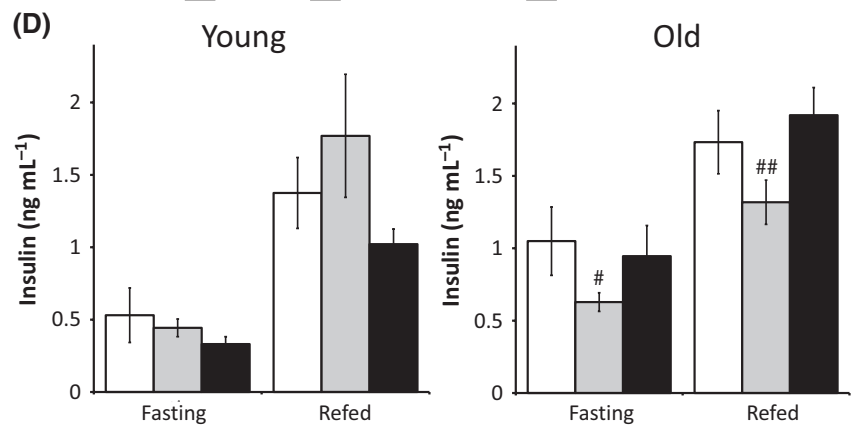

(E)

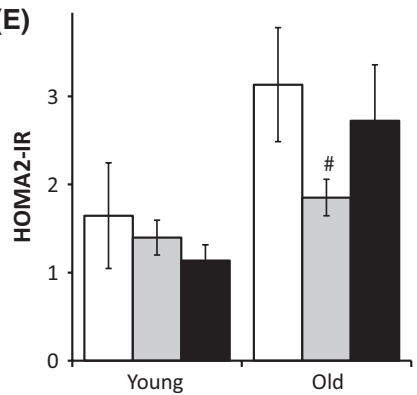

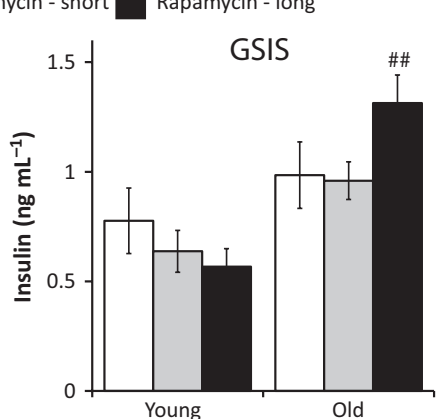

(F)

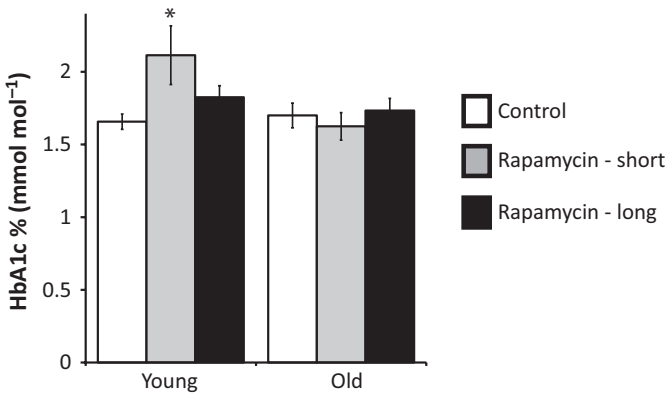

Fig. 2 Rapamycin treatment does not affect insulin resistance or insulin levels in HET3 mice. Insulin tolerance was tested 1 week after glucose tolerance in the same mice: (A) young (6-month-old) and (B) old (21-month-old) mice treated with rapamycin for 4 weeks or 3.3 months. (A: *P<0.05 vs. control mice, two tailed t-test.) ( $-\mathrm{D}$ ) Mice were fasted overnight and then refed for $4 \mathrm{~h}$, and $(C)$ blood glucose and $(D)$ insulin were measured in both feeding states $(C: * * P<0.01$, $P=0.059$, \#\# $P=0.073$ vs. control mice, two tailed $t$-test; $\mathrm{D}:{ }^{\#} P=0.16,{ }^{\# \#} P=0.173$ vs. control mice, two tailed $t$-test). (E) Left: HOMA-IR was calculated using the fasting glucose and insulin data in $C$ and D. Right: Glucose-stimulated insulin secretion (GSIS) was measured by fasting mice overnight, injecting $1 \mathrm{~g} \mathrm{~kg}^{-1} \mathrm{glucose}$, and collecting serum 15 min postinjection

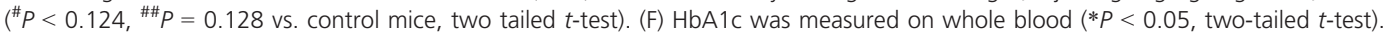


glucose injection, and old mice treated with rapamycin for 3 months had a trend toward hyperinsulinemia. However, insulin levels in several experimental groups are inappropriately normal despite significantly elevated glucose levels, suggesting that rapamycin may have an impact on beta cell function (Fig. 2C,D).

Finally, we used the fasted blood glucose and insulin levels to calculate insulin resistance using homeostasis model assessment (HOMA2-IR) (Levy et al., 1998). The HOMA2-IR model was derived empirically from human insulin-glucose clamp data, but is equally useful as a surrogate measure of insulin resistance in mice (Mather, 2009). We observed a trend toward decreased insulin resistance in old mice treated with rapamycin for a short period of time $(P=0.073$, two-tailed $t$-test) and did not find increased insulin resistance in any rapamycin-treated mice (Fig. 2E). To determine the cumulative effect of hepatic insulin resistance on blood glucose levels over time, we measured the level of glycated hemoglobin $(\mathrm{HbA} 1 \mathrm{c})$. While there was a significant increase in $\mathrm{HbA} 1 \mathrm{c}$ in young mice treated with rapamycin for 3 weeks, there was no increase in $\mathrm{HbA} 1 \mathrm{C}$ observed in young mice treated with rapamycin for 3 months, or in old mice treated with rapamycin (Fig. 2F).

As these data suggest the surprising result that HET3 mice treated with dietary rapamycin for a prolonged time do not have a defect in insulin tolerance and do not experience a major increase in average blood glucose levels, we examined the effect of rapamycin on male and female HET3 mice in an independent cohort of mice treated with rapamycin starting at approximately 20 months of age for 6-8 months. We found no significant difference in insulin resistance in rapamycin-treated mice relative to age-matched controls (Fig. 3A, $B)$. While fasting blood glucose in female mice treated with rapamycin was similar to age-matched controls, male mice treated with rapamycin for 20 months were hypoglycemic during fasting (Fig. 3C). Neither males nor females had altered HbA1c levels relative to age-matched controls (Fig. 3D). We also examined the serum level of fructosamine, which, like $\mathrm{HbA} 1 \mathrm{c}$, reflects average serum glucose levels and found that fructosamine levels were decreased in rapamycin-treated female mice and increased in male mice compared with old controls (Fig. 3D).

\section{Discussion}

In genetically heterogeneous HET3 mice, dietary rapamycin increased life span when treatment was started at 20 months of age (Harrison et al., 2009) or 9 months of age (Miller et al., 2011). However, in young C57BL/6 and BALB/C inbred mice, rapamycin treatment decreased glucose tolerance and increased insulin resistance (Cunningham et al., 2007; Lamming et al., 2012), a surprising result which runs counter to both intuition and a significant correlation between insulin sensitivity and longevity. To clarify, we analyzed both short-term and long-term treatment with dietary rapamycin in young and old HET3 mice.

Concurring with our previous study in C57BL/6 mice, HET3 mice treated with rapamycin were hyperglycemic after refeeding (Fig. 2C) and when young and treated with rapamycin for 3 months, also displayed fasting hyperglycemia. Hyperglycemia
(A)

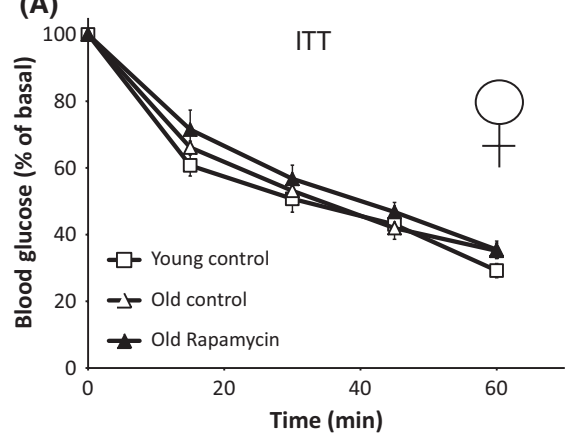

(C)

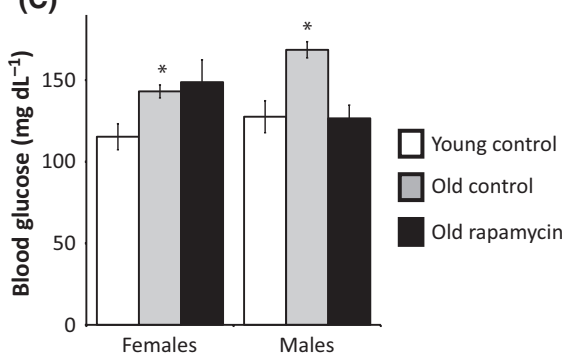

(B)
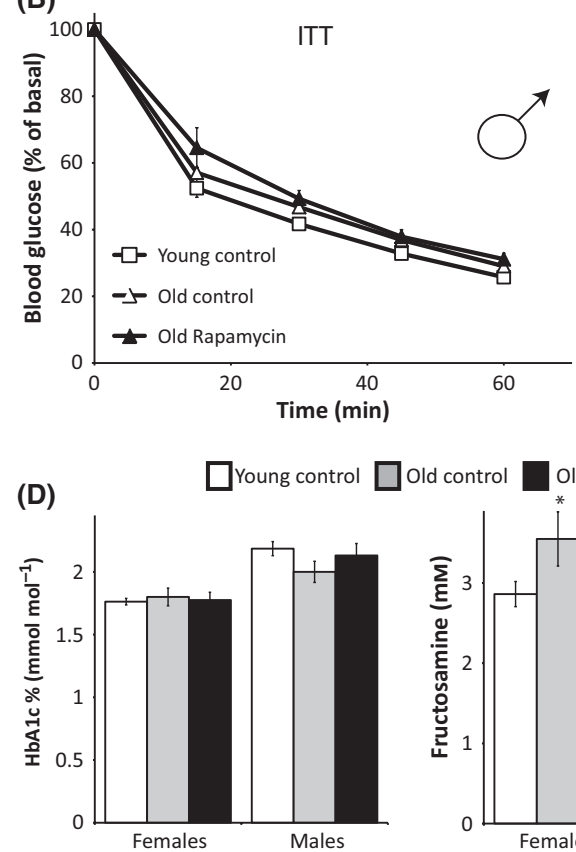

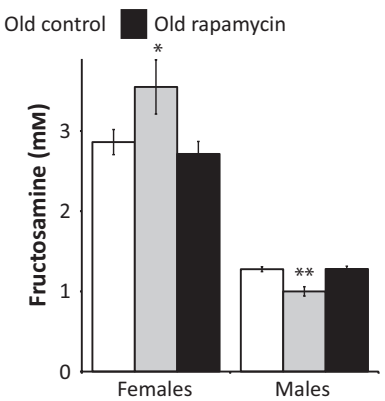

Fig. 3 Rapamycin treatment does not negatively impact insulin tolerance or HbA1c levels in older HET3 mice treated for $5-7$ months. Insulin tolerance test was performed on (A) female and (B) male HET3 mice. Average ages for female (F) and male (M) mice are given in months: young control (F, 13.0; $M$, 11.6), old control (F, 27.5; $M$, 25.7); old mice on rapamycin from 20 months of age (F, 27.4; M, 25.4). (C) Old male and female controls have fasting hyperglycemia relative to young controls and (female only) relative to rapamycin-treated old mice ( $* P<0.006$, two-tailed $t$-test). (D) HbA1c and fructosamine were measured in the same mice. There was no effect of age or rapamycin on $\mathrm{HbA} 1 \mathrm{c}$ levels; however, fructosamine was lower in old female mice treated with rapamycin compared with controls (** $P<0.01)$ and trended higher in old males receiving rapamycin $(* P<0.06$, two-tailed $t$-tests). 
and glucose intolerance are the first symptoms of type 2 diabetes in humans, but changes in $\mathrm{HbA} 1 \mathrm{c}$ were observed only in young mice treated for a short period of time compared with age-matched controls (Figs 2F and 3D, discussed below). The phenotype of HET3 mice treated with rapamycin for a prolonged time is strikingly similar to that of mice lacking the hepatic Rictor gene, which exhibit glucose and pyruvate intolerance but display an essentially normal response to an insulin tolerance test (Lamming et al., 2012). Just as with HET3 mice treated with rapamycin, mice lacking Rictor display hyperglycemia and hyperinsulinemia following refeeding. While we found that the decrease in glucose tolerance in rapamycin-treated C57BL/6 mice was largely attributable to the disruption of hepatic mTORC2, Blattler et al. (2012) have proposed an alternative mechanism by which rapamycin can decrease the expression of insulin signaling components in skeletal muscle. It will be important to dissect the relative contributions of these two mechanisms to the effects of dietary rapamycin in both young and old HET3 mice in future studies.

To determine whether the defective glucose homeostasis in the HET3 mice leads to increased average blood glucose levels, we assayed glycated hemoglobin ( $\mathrm{HbA} 1 \mathrm{c})$. Surprisingly, while there was an increase in $\mathrm{HbA1c}$ levels in young mice treated with rapamycin for 3 weeks, we observed no changes in young mice treated for 3 months or in older mice regardless of treatment time (Figs $2 \mathrm{~F}$ and 3D). This suggests that during the course of the initial ITP life span study using 20-month-old mice, rapamycin-treated HET3 mice did not experience higher average blood glucose levels. Even mice used in the follow-up life span study, which initiated treatment at 9 months of age, probably experienced little or no increased blood glucose. These data may resolve the seeming paradox of rapamycin promoting life span in the context of glucose intolerance and suggest that impaired glucose tolerance, but not necessarily systemic insulin resistance, is compatible with increased longevity. These data may resolve the seeming paradox that rapamycin increases life span in the context of glucose intolerance. They suggest that impaired glucose tolerance, but not necessarily systemic insulin resistance, is compatible with increased longevity. This contradicts the common conception that impaired glucose tolerance will negatively affect mammalian life span.

While rapamycin treatment of inbred mouse and rat strains results in clear insulin resistance, HET3 mice on the ITP rapamycin diet do not exhibit overt defects in an insulin tolerance test and do not display insulin resistance as calculated by the HOMA-IR method (Fig 2). It remains possible that HET3 mice on a rapamycin diet exhibit a smaller degree of insulin resistance that could be revealed with the aid of insulin-glucose clamps in a future study.

An important question raised by this study is the applicability of the rapamycin life span study to the clinic. Rapamycin treatment of transplant patients leads to hyperglycemia and insulin resistance, with an increased risk of diabetes (Johnston et al., 2008), but the effect of rapamycin on healthy human adults has not been extensively analyzed. C57BL/6 mice, which become insulin resistant when treated with rapamycin, may therefore more closely mimic the clinical experience of transplant patients than the insulin-sensitive HET3 mice. Of course, HET3 mice better model a genetically diverse, healthy human population, and using mice of mixed background ensures that results are not observed due to strain-specific lesions. Our findings highlight the importance of genetic background in biomedical research. Finally, rapamycin may extend life span in aged C57BL/6 mice despite the negative impact on insulin sensitivity; a recent small study observed a decreased mortality rate in 22 - to 24-month-old C57BL/6 mice injected for 6 weeks with rapamycin (Chen et al., 2009). Subsequent efforts should focus on determining the effects of strain background and delivery method on the ultimate effects of rapamycin on longevity.

\section{Experimental procedures}

\section{Animals}

$\mathrm{BALB} / \mathrm{CJ} \times \mathrm{C} 57 \mathrm{BL} / 6 \mathrm{~J}$ F1 female mice $\left(\mathrm{CB} 6 \mathrm{~F} 1 / \mathrm{J}, \quad J A X^{\circledR}\right.$ stock \# 100007) and $\mathrm{C} 3 \mathrm{H} / \mathrm{HeJ} \times \mathrm{DBA} / 2 \mathrm{~J} F 1$ male mice (C3D2F1/J, JAX ${ }^{\circledR}$ stock \# 100004) were obtained from The Jackson Laboratory and bred to produce heterogeneous HET3 F2 mice, a reproducible genetic background. The Jackson Laboratory is fully accredited by the American Association for Accreditation of Laboratory Animal Care, and all studies were conducted under a protocol approved by its the Animal Care and Use Committee (ACUC). Mice were produced and maintained in double-sided plastic cages in isolated rooms under positive pressure with high efficiency particulate airfiltered air at room temperature $\left(24 \pm 1^{\circ} \mathrm{C}\right)$, lighted from 6 am to $6 \mathrm{pm}$. After weaning, mice were housed four per side with others of the same sex and treatment. Results of quarterly screening for pathogens and other microorganisms at The Jackson Laboratory are provided online at http://jaxmice.jax.org/genetichealth/index. html. Mice in this study were housed in room D1, which did not have any pathogens for which The Jackson Laboratory routinely tests. We did not have to censor data due to ill health or loss of mice at The Jackson Laboratory or at MIT. During the studies at MIT, mice in this study were housed as sole occupants of a secondstage quarantine room and were not monitored for pathogens during the course of the experiment. The diet was the same as at The Jackson Laboratory. All experiments were approved by the Institutional Animal Care and Use Committees at MIT and were performed under the supervision of the MIT Department of Comparative Medicine.

\section{Rapamycin treatment protocol}

Fourteen ppm rapamycin was encapsulated in chow, LabDiet ${ }^{\circledR} 5 \mathrm{LG} 6$ (PMI Nutrition International, Bentwood, MO, USA); this is detailed by Harrison et al. (2009). Age-matched control mice were fed a 5 LG6 control diet without rapamycin. Young ( 6 months old) and old (21 months old) mice were fed rapamycin for either 3 weeks or 3 months. Three weeks is similar to conditions in our previous studies using C57BL/6J mice (Lamming et al., 2012); 3 months is more reflective of conditions in the rapamycin study in the ITP (Harrison et al., 2009; Miller et al., 2011). Rapamycin was fed to the treated mice throughout these experiments. The first assay done in the mice was glucose tolerance, insulin tolerance was tested a week later, and pyruvate tolerance 2 weeks later. 
Tolerance tests and assays

Glucose, insulin, and pyruvate

Glucose, insulin, and pyruvate tolerance tests were performed by fasting the mice overnight for $16 \mathrm{~h}$ and then injecting either glucose $\left(1 \mathrm{~g} \mathrm{~kg}^{-1}\right)$, insulin $\left(0.75 \mathrm{U} \mathrm{kg}{ }^{-1}\right)$, or pyruvate $\left(2 \mathrm{~g} \mathrm{~kg}^{-1}\right)$ intraperitoneally as previously described (Lamming et al., 2012). Glucose measurements were performed at the specific time points indicated using a Bayer Contour blood glucose meter and test strips. For glucose-stimulated insulin secretion assays, mice were fasted overnight and injected with $1 \mathrm{~g} \mathrm{~kg}^{-1}$ glucose; 15 min after injection, $50 \mu \mathrm{L}$ of blood was collected in a heparinized tube. For fasting and refed blood glucose levels, mice were fasted overnight, and blood glucose was determined as above. $50 \mu \mathrm{L}$ of blood was collected in heparinized tubes, and mice were refed for $4 \mathrm{~h}$. Blood glucose levels were again determined, and another $50 \mu \mathrm{L}$ of blood was collected in heparinized tubes. Blood samples were spun at $5400 \mathrm{~g}$ for $10 \mathrm{~min}$ in a refrigerated centrifuge, and plasma was collected and kept at $-80{ }^{\circ} \mathrm{C}$ until analysis. Plasma insulin levels were determined by ELISA, performed by the U. Penn Diabetes Research Center core facility.

\section{Glycated hemoglobin (HbA1C)}

Hemoglobin A1c was measured as detailed in the Beckman Coulter SYNCHRON Systems Chemistry information sheet A18497 (https:// www.beckmancoulter.com/wsrportal/techdocs?docname=/cis/A18 497/AG/EN_HbA1c.pdf). Total hemoglobin concentration was measured by a standard colorimetric method, while hemoglobin A1C antibodies combine with hemoglobin A1c from the sample to form soluble antigen-antibody complexes. Polyhaptens from the reagent then bind with the excess antibodies, and the resulting agglutinated complex is measured turbidimetrically by absorbance at 340 nanometers. These are used to express hemoglobin A1c concentration as a percentage of total hemoglobin.

\section{Fructosamine}

Fructosamine was measured as detailed in the Polymedco information sheet, using the enzymatic assay of the Poly-Chem System (http:// www.polymedco.com/chemistries-special-chemistries-pages-111. php). To measure fructosamine, a proteinase digests the glycated protein to yield glycated protein fragments. Ketamine oxidase oxidizes the ketamine bond of the glycated protein fragments. As a result, hydrogen peroxide is released and measured in a colorimetric Trinder end-point reaction.

\section{Statistical analysis and HOMA2-IR}

All comparisons were performed using two-tailed $t$-tests. Area under the curve was calculated using Prism (GraphPad Software). HOMA2-IR was calculated using the fasted blood glucose levels and fasting insulin levels of mice fasted overnight for $16 \mathrm{~h}$, using the HOMA2-IR calculator (http://www.dtu.ox.ac.uk/homacalculator/ index.php) (Wallace et al., 2004).

\section{Acknowledgments}

We would like to thank all the members of the Sabatini lab, especially P. Katajisto, A. Hutchins, M. Pacold, and W. Combs for help with protocols, reagents, and advice. We also especially thank Adam Salmon for valuable advice, consultation, and for critical reading of the manuscript. At the Baur lab, we thank Mi (Michelle) Kwon for excellent technical support. At the Harrison lab, we thank Vicki Ingalls, Pam Krason, and Nelson Durgin for expert animal production and care, Mike Astle for expert supervision, and Joanne Currer for editing this manuscript. This project was funded in part by grants from the NIH, NIA (AG 035860 and AG 022308), NCI (CA 129105), a Julie Martin Mid-Career Award in Aging Research from the American Federation of Aging Research (AFAR) to DMS, and a Research Grant from AFAR and a Bingham Trust Pilot Award from Penn's Institute on Aging to JAB. DWL is supported by a K99/R00 award from the NIH/ NIA (1K99AG041765-01A1). LY is supported by a Postdoctoral Fellowship from the American Heart Association, 7600031. Core services were provided by Penn's DERC (P30DK19525) and by The Jackson Laboratory's cancer core grant (P30 CA034196). DMS is an Investigator of the Howard Hughes Medical Institute.

\section{References}

Baur JA, Pearson KJ, Price NL, Jamieson HA, Lerin C, Kalra A, Prabhu VV, Allard JS, Lopez-Lluch G, Lewis K, Pistell PJ, Poosala S, Becker KG, Boss O, Gwinn D, Wang M, Ramaswamy S, Fishbein KW, Spencer RG, Lakatta EG, Le Couteur D, Shaw RJ, Navas P, Puigserver P, Ingram DK, de Cabo R, Sinclair DA (2006) Resveratrol improves health and survival of mice on a high-calorie diet. Nature 444, 337 342.

Blattler SM, Cunningham JT, Verdeguer F, Chim H, Haas W, Liu H, Romanino K, Ruegg MA, Gygi SP, Shi Y, Puigserver P (2012) Yin Yang 1 deficiency in skeletal muscle protects against rapamycin-induced diabetic-like symptoms through activation of insulin/IGF signaling. Cell Metab. 15, 505-517.

Chen C, Liu Y, Zheng P (2009) mTOR regulation and therapeutic rejuvenation of aging hematopoietic stem cells. Sci. Signal 2, ra75.

Cunningham JT, Rodgers JT, Arlow DH, Vazquez F, Mootha VK, Puigserver P (2007) mTOR controls mitochondrial oxidative function through a YY1PGC-1alpha transcriptional complex. Nature 450, 736-740.

Dominici FP, Hauck S, Argentino DP, Bartke A, Turyn D (2002) Increased insulin sensitivity and upregulation of insulin receptor, insulin receptor substrate (IRS)-1 and IRS-2 in liver of Ames dwarf mice. J. Endocrinol. 173, 81-94.

Flurkey K, Astle CM, Harrison DE (2010) Life extension by diet restriction and $\mathrm{N}$-acetyl-L-cysteine in genetically heterogeneous mice. J. Gerontol. A Biol. Sci. Med. Sci. 65, 1275-1284.

Fok WC, Zhang Y, Salmon AB, Bhattacharya A, Gunda R, Jones D, Ward W, Fisher K, Richardson A, Perez VI (2012) Short-term treatment with rapamycin and dietary restriction have overlapping and distinctive effects in young mice. J. Gerontol. A Biol. Sci. Med. Sci. 68, 108-116.

Foukas LC, Bilanges B, Bettedi L, Pearce W, Ali K, Sancho S, Withers DJ, Vanhaesebroeck B (2013) Long-term p110alpha PI3K inactivation exerts a beneficial effect on metabolism. EMBO Mol. Med. 5, 563-571.

Harrison DE, Strong R, Sharp ZD, Nelson JF, Astle CM, Flurkey K, Nadon NL, Wilkinson JE, Frenkel K, Carter CS, Pahor M, Javors MA, Fernandez E, Miller RA (2009) Rapamycin fed late in life extends lifespan in genetically heterogeneous mice. Nature 460, 392-395.

Hogan P, Dall T, Nikolov P (2003) Economic costs of diabetes in the US in 2002. Diabetes Care 26, 917-932.

Houde VP, Brule S, Festuccia WT, Blanchard PG, Bellmann K, Deshaies Y, Marette A (2010) Chronic rapamycin treatment causes glucose intolerance and hyperlipidemia by upregulating hepatic gluconeogenesis and impairing lipid deposition in adipose tissue. Diabetes 59, 1338-1348.

Johnston O, Rose CL, Webster AC, Gill JS (2008) Sirolimus is associated with new-onset diabetes in kidney transplant recipients. J. Am. Soc. Nephrol. 19, 1411-1418. 
Lamming DW, Ye L, Katajisto P, Goncalves MD, Saitoh M, Stevens DM, Davis JG, Salmon AB, Richardson A, Ahima RS, Guertin DA, Sabatini DM, Baur JA (2012) Rapamycin-induced insulin resistance is mediated by mTORC2 loss and uncoupled from longevity. Science 335, 1638-1643.

Levy JC, Matthews DR, Hermans MP (1998) Correct homeostasis model assessment (HOMA) evaluation uses the computer program. Diabetes Care $\mathbf{2 1}$ 2191-2192.

Mather K (2009) Surrogate measures of insulin resistance: of rats, mice, and men. Am. J. Physiol. Endocrinol. Metab. 296, E398-E399.

Miller RA, Harrison DE, Astle CM, Baur JA, Boyd AR, de Cabo R, Fernandez E, Flurkey K, Javors MA, Nelson JF, Orihuela CJ, Pletcher S, Sharp ZD, Sinclair D, Starnes JW, Wilkinson JE, Nadon NL, Strong R (2011) Rapamycin, but not resveratrol or simvastatin, extends life span of genetically heterogeneous mice. J. Gerontol. A Biol. Sci. Med. Sci. 66, 191-201.

Sarbassov DD, Ali SM, Sengupta S, Sheen JH, Hsu PP, Bagley AF, Markhard AL, Sabatini DM (2006) Prolonged rapamycin treatment inhibits mTORC2 assembly and Akt/PKB. Mol. Cell 22, 159-168.

Selman C, Lingard S, Choudhury Al, Batterham RL, Claret $M$, Clements $M$, Ramadani F, Okkenhaug K, Schuster E, Blanc E, Piper MD, Al-Qassab H,
Speakman JR, Carmignac D, Robinson IC, Thornton JM, Gems D, Partridge L, Withers DJ (2008) Evidence for lifespan extension and delayed age-related biomarkers in insulin receptor substrate 1 null mice. FASEB J. 22, 807-818.

Selman C, Tullet JM, Wieser D, Irvine E, Lingard SJ, Choudhury Al, Claret M, Al-Qassab H, Carmignac D, Ramadani F, Woods A, Robinson IC, Schuster E, Batterham RL, Kozma SC, Thomas G, Carling D, Okkenhaug K, Thornton JM, Partridge L, Gems D, Withers DJ (2009) Ribosomal protein S6 kinase 1 signaling regulates mammalian life span. Science 326, 140-144.

Teutonico A, Schena PF, Di Paolo S (2005) Glucose metabolism in renal transplant recipients: effect of calcineurin inhibitor withdrawal and conversion to sirolimus. J. Am. Soc. Nephrol. 16, 3128-3135.

Wallace TM, Levy JC, Matthews DR (2004) Use and abuse of HOMA modeling. Diabetes Care 27, 1487-1495.

Yang SB, Lee HY, Young DM, Tien AC, Rowson-Baldwin A, Shu YY, Jan YN, Jan LY (2012) Rapamycin induces glucose intolerance in mice by reducing islet mass, insulin content, and insulin sensitivity. J. Mol. Med. (Berl) 90, 575-585 